\title{
Preface: The Review of Philosophy and Psychology
}

\author{
Dario Taraborelli • Roberto Casati • Paul Egré • Christophe Heintz
}

Published online: 20 February 2010

(C) Springer Science+Business Media B.V. 2010

In the 1876 introduction to the first issue of Mind, its then editor George C. Robertson set among the goals of the journal the question of deciding on the possibility of psychology as a science, noting that:

[e]ither psychology would in time pass with general consent into the company of the sciences, or the hollowness of its pretensions would be plainly revealed (Robertson 1876).

The journal - soon to become the most respected organ of the analytic traditiondefined itself in its early years as "the first English journal devoted to Psychology and Philosophy" and displayed in its masthead: "A Quarterly Review of Philosophy and Psychology", a subtitle only to be abandoned in 1972 (Staley 2009). Today, whether contemporary psychology can be measured by scientific standards is no longer a question in need of an answer. Yet, what contribution philosophical methods and theoretical analysis can bring to the understanding of cognition is still very much an open question - which the Review of Philosophy and Psychology aims to tackle.

Over the last decades, the scientific study of the mind has become a vast new territory of philosophical inquiry with the emergence of cognitive science as a leading research paradigm; a revolution to which philosophers substantially

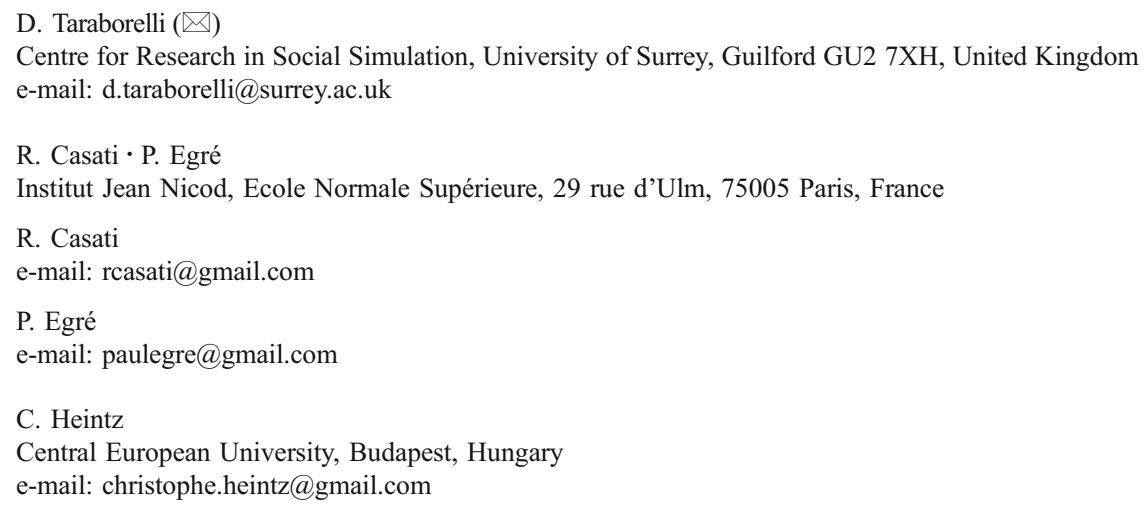


contributed. This revolution opened up new possibilities for the cross-pollination of philosophy and the sciences of the mind. Philosophers and cognitive scientists are jointly defining the scope, theoretical foundations and agenda of cross-disciplinary research on the mind/brain. Prominent cases of debates in which this happened include, among others: the debate on empiricism and the innate mind, the neural bases of consciousness, the cognitive underpinning of rationality, the interface between culture and cognition. Moreover, empirical results keep providing naturalised grounds for philosophical inquiry. Identifying the conceptual landmarks and helping consolidate the geography of cognitive science is the goal that this journal aims to achieve.

The Review of Philosophy and Psychology was originally published as the European Review of Philosophy, a series in analytic philosophy of mind founded in Geneva in the mid 1990s. The new journal builds on the legacy of its predecessor. Its ambition is to offer both a critical and foundational contribution to the current debate in cognitive science, as illustrated by the different angles of the forthcoming issues.

- The journal hosts foundational debates on concepts and notions that ground experimental research: Issue 1 brings together psychologists and philosophers in redefining the notion of an "auditory object" and clarifying its implications for empirical research (Bullot and Egré 2010).

- It also aims to bridge distinct research programmes across philosophy and cognitive science: Issue 2 sets the scope of current investigations on folk epistemology, offering an overview of research at the interface of psychology (in particular the most recent developments on the ontogeny of trust) and epistemology (Heintz et al. 2010).

- Finally, it fosters the debate on common methodological challenges for philosophers and behavioural and social scientists: Issues 3 and 4 are devoted to critically assess the application of empirical methods to philosophical enquiry, by reviewing a range of case studies and methodological proposals in the area of experimental philosophy (Machery et al. 2010).

Faithful to the vision of its predecessor, the Review of Philosophy and Psychology aims to achieve these goals by hosting targeted discussions in the format of thematic issues. Each issue features articles by invited authors as well as articles solicited through a thematic call for papers and combines philosophical contributions with articles discussing on the theoretical implications of empirical research. It also publishes unsolicited articles that fall within the scope of the journal.

The mission of the RPP is supported by a world-class board of scientific advisors from a variety of fields that cover the broad disciplinary scope of the journal, including philosophy; linguistics; cognitive and developmental psychology; cognitive anthropology, ethology and neuroscience. Two scientific societies have been instrumental in promoting research in the field of the journal, the Society for Philosophy and Psychology (SPP) and its younger European counterpart, the European Society for Philosophy and Psychology (ESPP). The journal is strengthening its ties with these societies (ESPP in particular), that represent an essential part of its readership and provides researchers in the field with a dedicated outlet for their best research. 


\section{References}

Robertson, G.C. 1876. I-Prefatory words. Mind 1(1)

Staley, T.H. 2009. The journal Mind in its early years, 1876-1920: An introduction. Journal of the History of Ideas 70(2): 259-263.

Bullot, N.J., and P. Egré. 2010. Editorial: Objects and Sound Perception. Review of Philosophy and Psychology 1(1)

Heintz, C., N. Pouscoulous, and D. Taraborelli. 2010. Editorial: Folk Epistemology. Review of Philosophy and Psychology 1(2)

Machery, E., T. Lombrozo, and J. Knobe. 2010. Editorial: Psychology and Experimental Philosophy. Review of Philosophy and Psychology 1: 3-4. 